\title{
Implications of Capacitor Voltage Imbalance on the Operation of the Semi-Full-Bridge Submodule
}

\author{
Stefanie Heinig, Keijo Jacobs, Kalle Ilves, Staffan Norrga, and Hans-Peter Nee \\ KTH Royal Institute of Technology, EES/EPE \\ Teknikringen 35 \\ SE-100 44 Stockholm, Sweden \\ Email: sheinig@kth.se
}

\section{Acknowledgments}

Stefanie Heinig's research project has received funding from the Elektra program of the Swedish Energy Research Centre. Keijo Jacobs' research project is funded by the Swedish Centre for Smart Grids and Energy Storage (SweGRIDS). The authors would also like to acknowledge ABB Corporate Research Center (SECRC) for their valuable support.

\section{Keywords}

$\ll$ Multilevel converters $\gg, \ll$ Voltage source converter (VSC) $\gg, \ll H V D C \gg$

\begin{abstract}
An investigation of the voltage imbalance of the two capacitors of the semi-full-bridge submodule is performed. Since the capacitances are not exactly the same, there may be a difference between the capacitor voltages. The resulting current-spike when they are connected in parallel has been analyzed in a full-scale laboratory experiment.
\end{abstract}

\section{Introduction}

Two of the most important components of future high-voltage direct current (HVDC) supergrids are the power converters and dc circuit breakers [1,2]. During recent years viable candidate technologies for both these components have been proposed. In the case of power converters, the preferred converter is the modular multilevel converter (MMC), first presented by Marquardt et al. [3-6], and in the case of dc circuit breakers various hybrid electronic/mechanical concepts have been proposed [7]. Both the converter and the dc circuit breaker are costly and voluminous to such an extent that the introduction of HVDC supergrids may be delayed unless more cost-effective and less bulky solutions are found. The cost for circuit breakers could be reduced by using power converters that are able to control the dcside current in the event of a short circuit on the dc side, because dc circuit breakers may not have to be installed in every single node if such converters are used [8]. Submodule topologies that fulfill this criterion are full-bridge submodules and double-clamp submodules (DCS) [9].

Regarding the cost and volume of the power converter itself, it is found that a major contributor is the submodule capacitance $[10,11]$. Therefore, it is important to find submodule topologies which enable efficient handling of dc-side short circuits while having a minimum amount of submodule capacitance. One very interesting submodule topology from this point of view is the semi-full-bridge submodule, hereafter referred to as SFB submodule, presented in [12]. By using the possibility to insert negative voltages in the converter arms, it was shown that the ripple voltage across the submodule capacitor could be reduced by $59 \%$ compared to the case when only positive voltages were used. The same negative voltages are also necessary in order to set up a voltage against the ac-side voltage in case of a dc-side 
short circuit. Another interesting feature of this submodule topology is that the two submodule capacitors in the circuit can be connected either in series or in parallel when inserting positive voltages in the arm. In the latter case, the submodule capacitors can share the arm current such that the corresponding voltage deviation across the submodule capacitors is reduced by $50 \%$ during the particular conduction interval. The parallel connection may, however, also be problematic in case the two capacitor voltages are not equal. Due to the low impedance of the circuit, the voltage imbalance may give rise to excessive redistribution currents and subsequent oscillations between the two capacitors. This phenomenon is studied in detail in the present paper.

\section{Semi-full-bridge submodule operating principles}

The use of SFB instead of full-bridge submodules reduces the number of components in an MMC but still provides fault current limitation and bipolar voltage capability. The operation principles of the SFB and its energy fluctuation has been studied in [12]. The SFB can be considered as an extension of the DCS as presented in [9]. The main difference between the DCS and the SFB is that two diodes have been replaced by active switches which changes the function and operating principles of the submodule. The proposed SFB submodule contains two capacitors which can be bypassed, connected in parallel, or in series. Thus, the SFB combines a half-bridge and a full-bridge submodule as shown in Fig. 1.

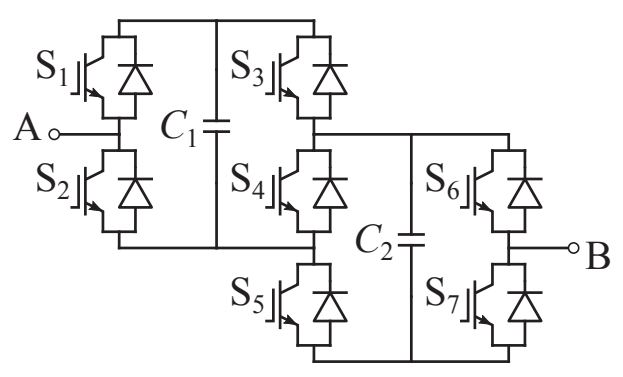

Fig. 1: Schematic diagram of the proposed semi-full-bridge submodule.

The two for this investigation relevant switching states of the SFB are illustrated in Fig. 2 where devices that are turned off are shown in gray color.
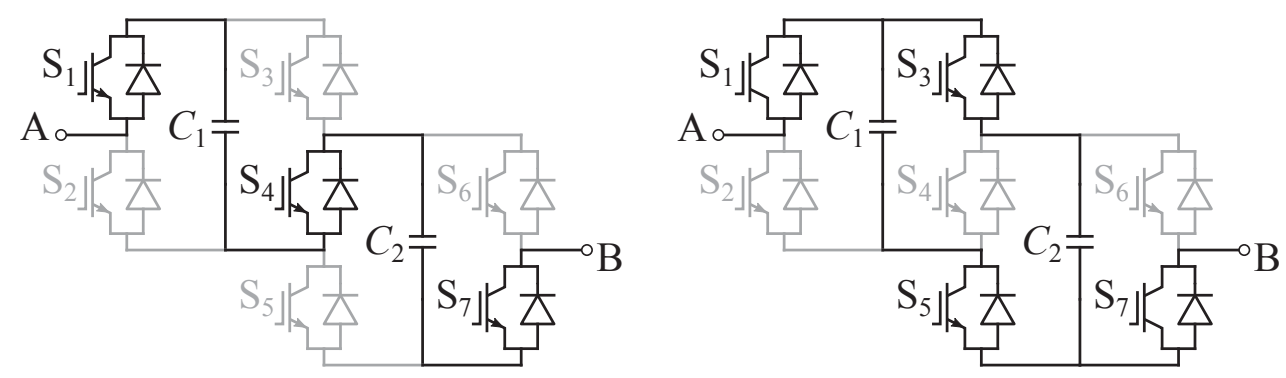

Fig. 2: Switching states when the capacitors are inserted in series (left) and in parallel (right).

\section{Capacitor voltage imbalance}

In general, it has to be assumed that the capacitance values of $C_{1}$ and $C_{2}$ differ slightly due to manufacturing tolerances. After some time in operation the capacitance of film capacitors may also have decreased due to their self-healing or clearing functionality. At the position of a local defect, a small part of the stored energy punctures the film and evaporates the thin metal layer. Hence, the local defect is disconnected similar to the function of a fuse [13]. Consequently, the two capacitors charge differently when they are inserted in series although both are subjected to the same arm current. This results in different voltages across $C_{1}$ and $C_{2}$ which has been analytically derived in [12]. This voltage difference is balanced out when the switching state is changed from series- to parallel-connection of the capacitors. 
When commutating from series to parallel insertion, attention has to be paid to blanking time and the transient current caused by the balancing of the capacitor voltages. The equivalent circuit during the transient is illustrated in Fig. 3. The main elements of this circuit are the two capacitances $C_{1}$ and $C_{2}$, the stray inductance $L_{\sigma}$, and the voltage drop due to the built-in potential of the IGBT $V_{\text {th,IGBT }}$ and diode $V_{\mathrm{th}, \mathrm{D}}$. Furthermore, the circuit comprises several resistive elements, namely the equivalent series resistance of the capacitors $E S R_{\mathrm{C}}$, the ON-state resistances of the IGBT $R_{\mathrm{IGBT}}$ and the diode $R_{\mathrm{D}}$ as well as the resistance of the busbars $R_{\text {bus }}$, which can be lumped into one resulting resistance

$$
R_{\text {res }}=2 E S R_{\mathrm{C}}+R_{\mathrm{IGBT}}+R_{\mathrm{D}}+R_{\mathrm{bus}} .
$$

All these elements together form a damped resonance circuit. It should be noted that the redistribution current $i_{\mathrm{rd}}$ is superimposed on the arm current during this process. The arm current divides almost equally among $C_{1}$ and $C_{2}$. Hence, the sum of both currents establishes the current direction in $S_{3}$ and $S_{5}$ which in turn determines whether IGBT or diode are conducting.

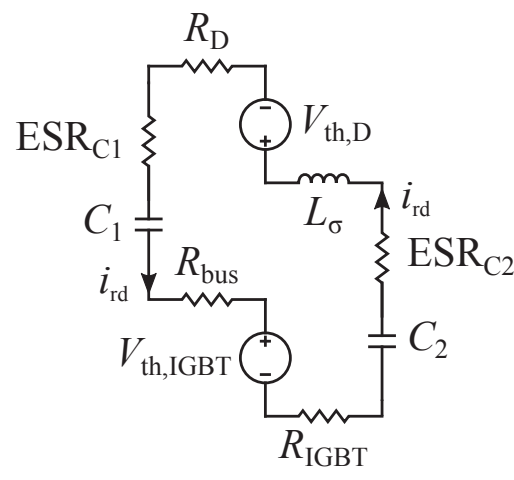

Fig. 3: Parasitic elements in the current path.

\section{Analytical calculation of the redistribution current}

If there is a voltage imbalance between the two capacitors in the SFB submodule there will be a current flowing when the capacitors are connected in parallel. This current redistributes charge from one capacitor to the other such that the two voltages become equal after a certain time. The analytical calculation of the current is based on the equivalent circuit presented in Fig. 3. It is assumed that both capacitors have the same capacitance $C$. Initially, the voltage of $C_{2}\left(V_{\mathrm{C} 2}\right)$ is higher than the voltage of $C_{1}\left(V_{\mathrm{C} 1}\right)$ such that at time zero the redistribution current is flowing as indicated in Fig. 3. The analysis of $i_{\text {rd }}$ reveals that

$$
i_{\mathrm{rd}}(t)=\frac{\Delta V_{\mathrm{C}}-V_{\mathrm{th}, \mathrm{D}}-V_{\mathrm{th}, \mathrm{IGBT}}}{\sqrt{2 \frac{L_{\sigma}}{C}-\frac{R_{\mathrm{res}}^{2}}{4}}} e^{-t / \tau} \sin (\omega t),
$$

where $\Delta V_{C}$ is given by the voltage difference between the capacitors

$$
\Delta V_{\mathrm{C}}=V_{\mathrm{C} 2}-V_{\mathrm{C} 1},
$$

with $\tau$ referring to the time constant that is given by

$$
\tau=\frac{2 L_{\sigma}}{R_{\text {res }}}
$$

and with $\omega$ describing the angular frequency that is given by

$$
\omega=\sqrt{\frac{2}{L_{\sigma} C}-\frac{1}{4}\left(\frac{R_{\mathrm{res}}}{L_{\sigma}}\right)^{2}} .
$$


It should be noted from (2) that it is not the total voltage level $V_{\mathrm{C} 1,2}$ that determines the redistribution current but only the voltage difference $\Delta V_{\mathrm{C}}$.

\section{Maximum differences in the capacitor voltages}

An analytical study has been conducted in order to evaluate the influence of various MMC parameters on the maximum differences in the capacitor voltages $\Delta V_{\mathrm{C} \text {,max }}$. The impact of the following parameters has been investigated: switching frequency, arm current $i_{\text {arm }}$ and phase angle $\phi$ as well as capacitance $C$ and its associated tolerances $k$.

Fig. 4 illustrates how the capacitor voltages develop during one fundamental cycle. Phase-shifted carrier pulsewidth modulation (PWM) has been chosen to define whether the capacitors of the SFB shall be inserted in series, parallel or bypassed.
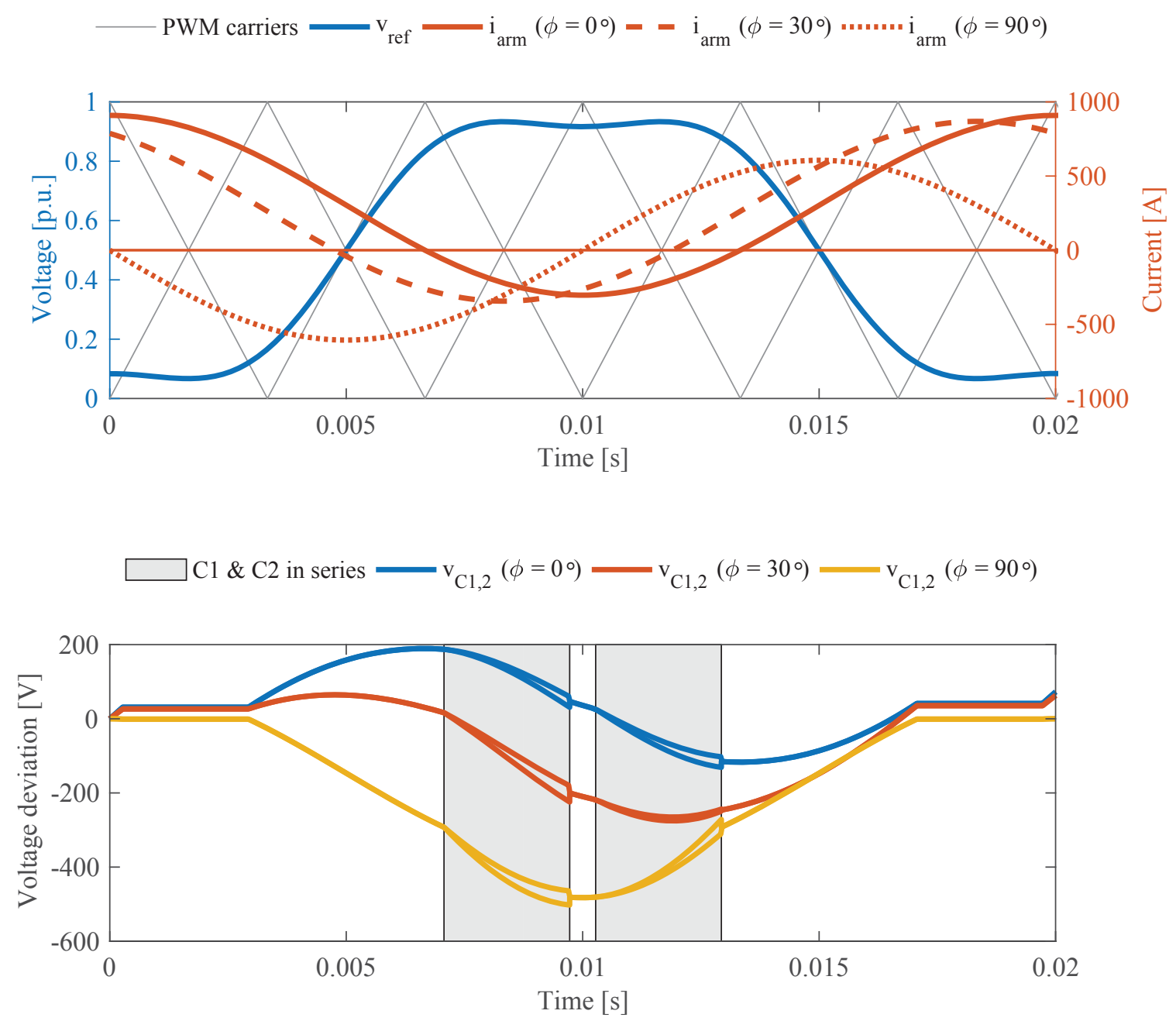

Fig. 4: MMC reference arm voltage and currents (top) and capacitor voltages (bottom).

The upper plot of Fig. 4 shows a reference arm voltage together with arm currents of different phase shift and two PWM carriers. The submodule switching state which generates negative terminal voltages is not part of this study since this state does not create any voltage imbalance among the two capacitors. Thus, only two instead of three carrier signals are needed for the modulation.

The corresponding capacitor voltages are shown in the lower plot of Fig. 4. Since a difference in the capacitance of the two capacitors has been assumed in this example $(k= \pm 10 \%)$, the capacitor voltages diverge during their series connection as highlighted in gray and are balanced out as soon as they are connected in parallel. It becomes clear that the longer the capacitors are connected in series the greater 
will be the resulting voltage difference, provided that the current is not changing direction during that time. Consequently, the voltage difference is inversely proportional to the carrier frequency. It can also be seen that the series connection of the capacitors coincides with low arm currents for active power transfer which is favorable in terms of $\Delta V_{\mathrm{C}, \max }$. The voltage difference becomes larger for increased phase angles because of higher arm currents during the series connection.

Fig. 5 presents the results of a parameter study for $\Delta V_{\mathrm{C}, \max }$. A reference case has been assumed, where $i_{\text {arm }}=900 A, \phi=0^{\circ}, C=4 \mathrm{mF}$ and $k= \pm 5 \%$. The two plots show a variation of the parameters $\phi$ and $k$ as a function of the switching frequency, while the remaining parameters are unchanged according to the reference case. The insets show a zoom to low switching frequencies since they are the most relevant in MMC-applications.

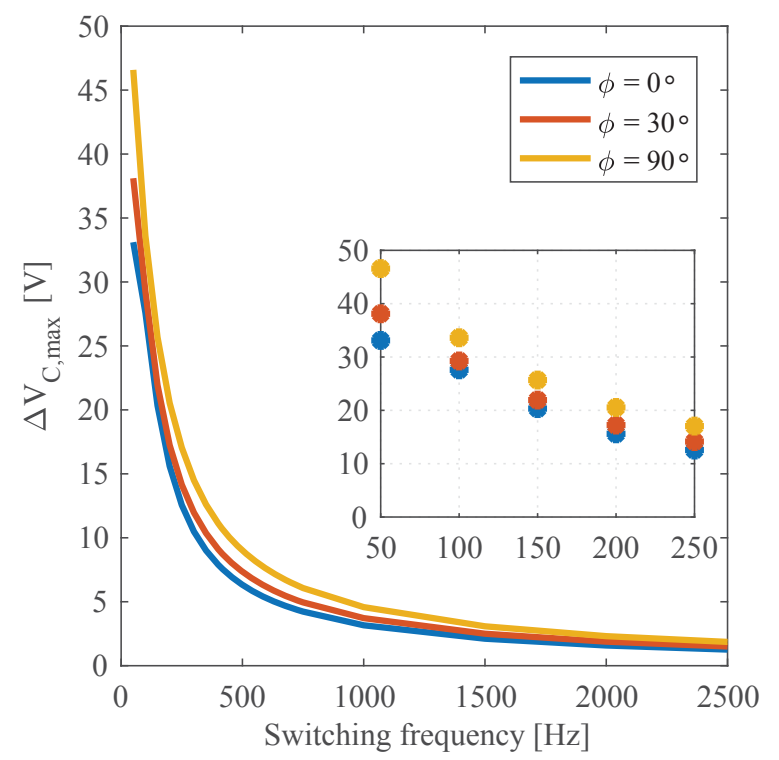

(a) Phase angle $\phi$

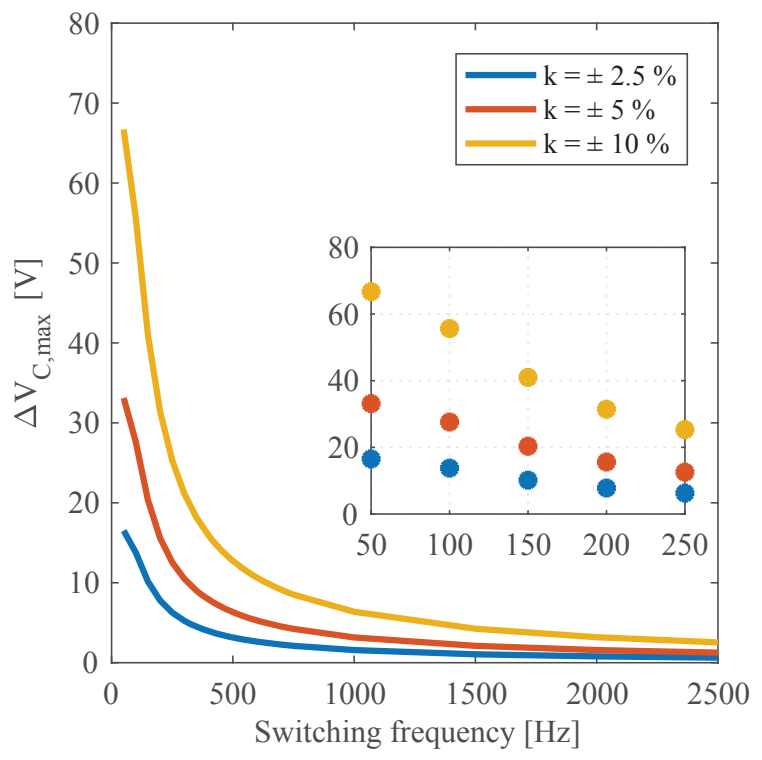

(b) Capacitance tolerance $k$

Fig. 5: Parameter study of the maximum differences in the capacitor voltages.

Both plots of Fig. 5 confirm the afore-stated inversely proportional relation between $\Delta V_{C, \max }$ and the switching frequency. It is also shown that the spread in $\Delta V_{\mathrm{C}, \max }$ is greatest for low switching frequencies and that it converges to a small value for high switching frequencies. Fig. 5a illustrates that the phase angle has a minor impact, in particular for small values of $\phi$, whereas the capacitance tolerance $k$ is critical concerning the amount of $\Delta V_{\mathrm{C}, \max }$ as shown in Fig. 5b. However, the (yellow) case where the capacitance of one capacitor is $10 \%$ above its nominal value and the capacitance of the other capacitor is $10 \%$ below its nominal value is highly unlikely. It can instead be reasonably assumed that the capacitances of a batch of new capacitors have similar values which are probably slightly above their rated values.

Fig. 5 shows that, except for the extreme case of $k= \pm 10 \%, \Delta V_{\mathrm{C}, \max }$ is lower than $30 \mathrm{~V}$ for switching frequencies greater than $100 \mathrm{~Hz}$ and lower than $20 \mathrm{~V}$ for switching frequencies greater than $150 \mathrm{~Hz}$. Immediately after parallelization of the capacitors, $\Delta V_{\mathrm{C}}$ will translate into a certain peak value of the redistribution current. This peak value can be calculated with (2) provided that the circuit parameters are known and should be kept within the safe operating area (SOA) of the employed semiconductor devices.

\section{Impact on the modulation of the semi-full-bridge submodule}

It is not obvious how a voltage imbalance between the two submodule capacitors should be handled in case the submodule should provide a positive voltage with two capacitors in parallel. If the total current, i.e. half of the arm current plus the redistribution current, is less than the maximum allowable current it may be possible to disregard the voltage imbalance.

In case the predicted current is too high there are primarily two options. The first option would be to insert only one of the capacitors until the two capacitor voltages are equal and then connect the other 
one in parallel. However, with this alternative one has to be careful and must ensure that the maximum current is lower than the rated current of the devices $S_{3}$ and $S_{5}$. Since it is possible to control the SFB in such a way that those IGBT modules are always conducting in parallel they could theoretically only be rated for half of the arm current. The second option would be to refrain from inserting the submodule until the expected current is within the SOA.

\section{Simulations and experimental results}

The behaviour of the redistribution current in the SFB submodule is validated by simulations with ideal components in LTspice ${ }^{\circledR}$. The equivalent circuit is shown in Fig. 6 which highlights the current path and direction in red color.

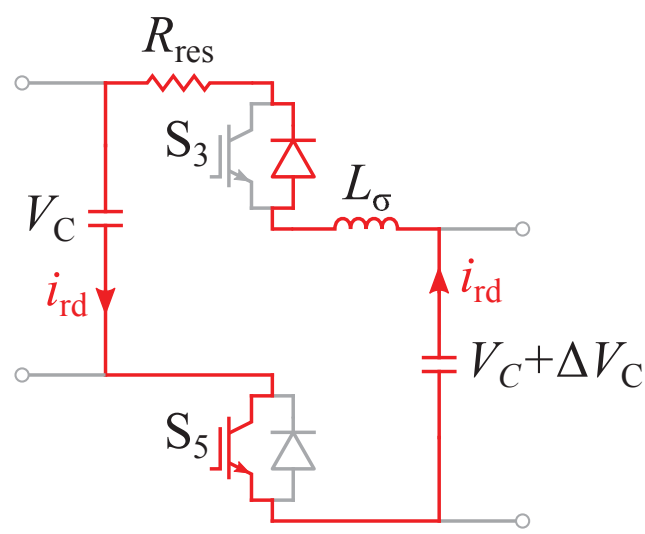

Fig. 6: Current path during the redistribution transient.

A voltage difference $\Delta V_{\mathrm{C}}$ of $40 \mathrm{~V}$ is assumed for the simulations. A first assessment of the overall resistance $R_{\text {res }}$ in the current path has been calculated based on measurements of the static characteristics of the actual semiconductor devices - 3300 V/800 A IGBT modules (MBN800E33E) manufactured by HITACHI - and copper busbars that are used in the experimental setup. The results show that the ONstate resistances of the IGBT modules, $R_{\mathrm{IGBT}}$ and $R_{\mathrm{D}}$, follow the $125^{\circ} \mathrm{C}$ characteristic given in their datasheet [14], and thus have a significant contribution to the overall resistance, whereas the measured resistance of the busbar system $R_{\text {bus }}$ is comparatively small.

The values used for $R_{\text {res }}$ and the stray inductance $L_{\sigma}$ in the simulation with LTspice ${ }^{\circledR}$ have been obtained from a curve fit of the current measurement data in MATLAB based on (2). Fig. 7 shows how the simulated redistribution current compares to the measurement. The fitted parameters are listed in Table I together with other specifications of the simulated circuit.

Table I: Simulation parameters

\begin{tabular}{ccc}
\hline Parameter & Symbol & Value \\
\hline Voltage difference & $\Delta V_{\mathrm{C}}$ & $40 \mathrm{~V}$ \\
Submodule capacitance & $C$ & $4 \mathrm{mF}$ \\
Stray inductance & $L_{\sigma}$ & $230 \mathrm{nH}$ \\
Equivalent resistance & $R_{\text {res }}$ & $7.85 \mathrm{~m} \Omega$ \\
Simulation time step & $\Delta t$ & $1 \mu \mathrm{s}$
\end{tabular}

The inductance value of $230 \mathrm{nH}$ coincides with calculations that have been performed according to [15] and on the basis of measurements for a high-power IGBT module [16]. Moreover, the resulting resistance of $7.85 \mathrm{~m} \Omega$ shows a good agreement with the previously determined measurement value. 


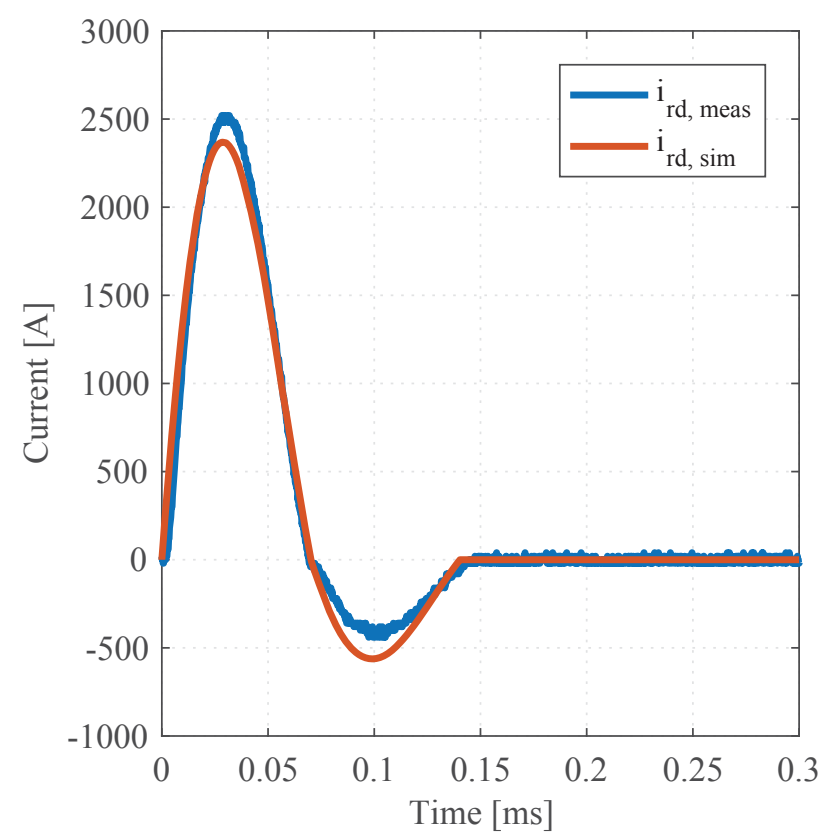

Fig. 7: Measurement data (blue) and simulation result (red) of the redistribution current.

Fig. 8 illustrates the charge balancing process between the two capacitors. Their voltages converge after $0.3 \mathrm{~ms}$ in the analytical calculation based on (2) but do not converge in the simulation. This is because LTspice ${ }^{\circledR}$ takes the forward voltage drop of the semiconductor devices into account which is not the case as regards the analytical calculation in MATLAB. The oscillation stops as soon as the remaining voltage difference is less than the combined forward voltage drop over IGBT and diode, which is approximately $3 V$ with the semiconductor module used.

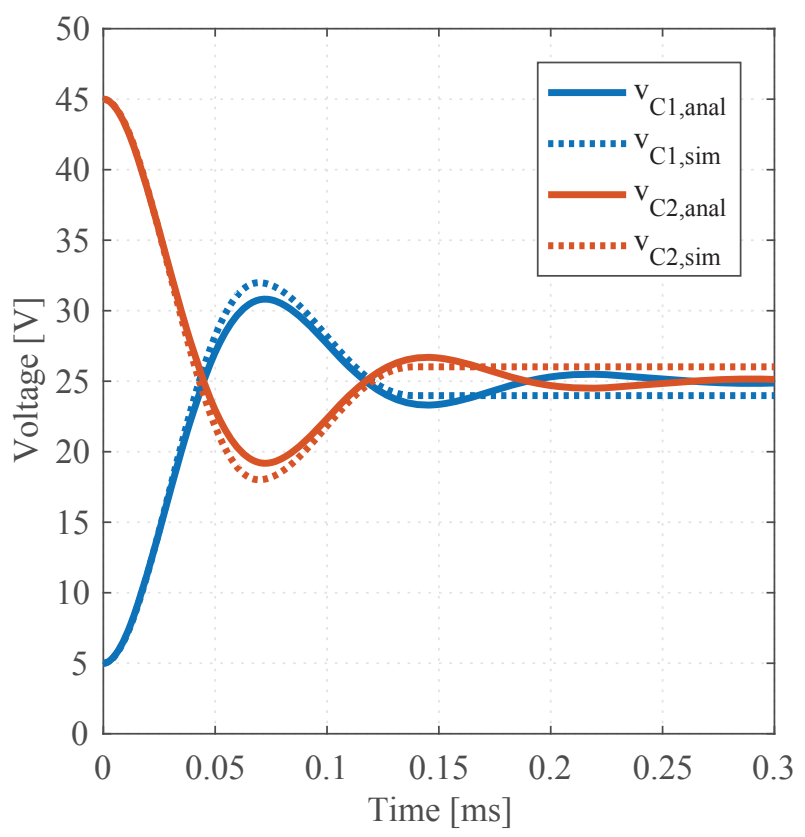

Fig. 8: Analytical calculation (solid) and simulation result (dashed) of the two capacitor voltages.

The described phenomenon of oscillating redistribution currents due to a voltage imbalance between the two capacitors of the SFB has been investigated experimentally on a full-scale prototype. A drawing of the setup is shown in Fig. 9a and a photograph of the realized implementation is presented in Fig. 9b. 


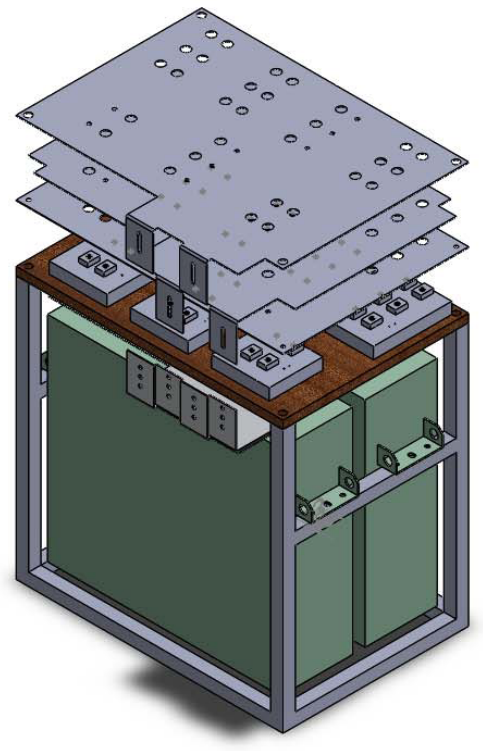

(a) Drawing of the SFB using SolidWorks ${ }^{\circledR}$.

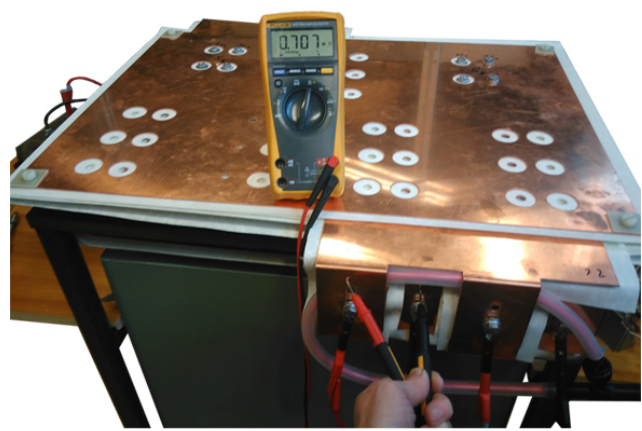

(b) Photograph of the busbars of the SFB.

Fig. 9: Experimental setup of the full-scale laboratory prototype of the SFB submodule.

The SFB submodule has been equipped with five 3300 V/1200 A (MBN1200E33E) and two 3300 V/ 800 A (MBN800E33E) IGBT modules, both types manufactured by HITACHI. However, for the tests conducted for this publication only two of the lower rated IGBT modules are needed. The two capacitors, manufactured by VISHAY, have a rated direct voltage of $2650 \mathrm{~V}$ and a nominal capacitance of $4 \mathrm{mF}$ with a tolerance of $\pm 5 \%$ as stated in the datasheet [17].

Fig. 10 shows current and voltage measurement results for the case of a small capacitor voltage difference of $\Delta V_{\mathrm{C}}=10 \mathrm{~V}$ and a more substantial difference of $\Delta V_{\mathrm{C}}=40 \mathrm{~V}$. The peak current amounts to $500 \mathrm{~A}$ and $2500 \mathrm{~A}$ respectively. The latter exceeds by far the repetitive peak collector current of the used $3300 \mathrm{~V} / 800 \mathrm{~A}$ IGBT module, which is $1600 \mathrm{~A}$ according to the datasheet [14]. Therefore, it can be concluded that capacitor voltage differences as high as $\Delta V_{\mathrm{C}}=40 \mathrm{~V}$ have to be strictly avoided.

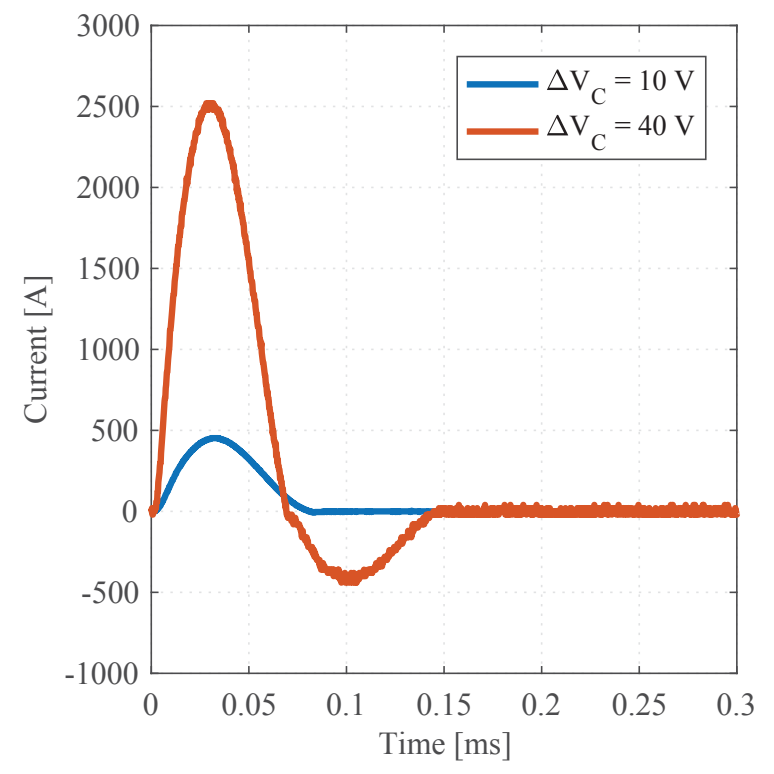

(a) Redistribution currents

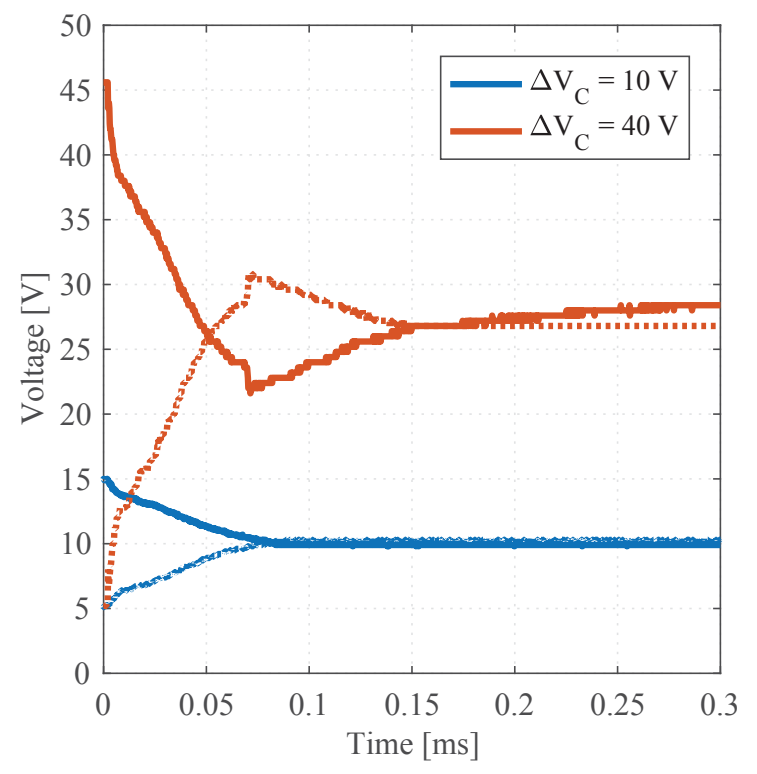

(b) Capacitor voltages

Fig. 10: Measurement results for two voltage differences $\Delta V_{\mathrm{C}}$ between the two capacitors. 


\section{Conclusion}

The SFB is a promising submodule topology for HVDC converters to be used in future dc-grids. It can generate negative terminal voltages in both current directions, which gives it the inherent capability of handling dc-side short circuits. Furthermore, the SFB also provides the possibility to operate the converter at increased modulation indices which can significantly reduce the energy storage requirements of the submodule capacitors. However, the SFB requires the parallel connection of capacitors during normal operation. If the capacitor voltages are not equal, the freedom of using all possible switching states might be limited and the paralleling can cause high oscillating redistribution currents. This has to be strictly avoided by implementing proper switching sequences and capacitor balancing strategies.

An analytical parameter study of the maximum differences in the capacitor voltages has been conducted. It turned out that the phase angle has only a minor impact, while the capacitance tolerance is critical. The results show that the amount of voltage difference should be no problem in most of the cases, except for those where a very high value of the capacitance tolerance has been assumed. Neglecting these unlikely cases, the maximum difference in the capacitor voltages is $20-30 \mathrm{~V}$ for switching frequencies in the range of $100-150 \mathrm{~Hz}$.

It can be concluded that it is recommended to minimize a potential mismatch between the two capacitances of one submodule. Since the capacitance is usually measured and documented by the manufacturer, the capacitors could be grouped in pairs in order to minimize the difference from the very beginning. In the opinion of the authors, the problem of a major mismatch between the capacitances might occur only after several years of operation due to different stresses and aging processes. It is important to be aware of this and to have safe control strategies at hand that can handle situations of that kind. Such stategies have not been studied in the context of this paper but are the subject of future research.

A full-scale prototype of the SFB submodule has been built in order to experimentally investigate and quantify the redistribution current due to an existing voltage imbalance between the two capacitors. The measurement data shows good agreement with the analytical calculations and simulation results. It could be demonstrated that the redistribution current peaks at $500 \mathrm{~A}$ for a capacitor voltage difference of $10 \mathrm{~V}$ and at $2500 \mathrm{~A}$ if the difference is $40 \mathrm{~V}$ before paralleling.

Moreover, it has been possible to identify the circuit parameters of a realistic SFB submodule implementation from the full-scale experimental setup. An important finding is that the circuit is highly damped and the oscillations cease quickly. Those parameters can be used in the analytical expression such that peak value and frequency of the redistribution current can be determined from measurements of the capacitor voltage difference. Based on this knowledge the submodule controller or the high-level control can decide how to handle the voltage imbalance.

\section{References}

[1] N. Ahmed, A. Haider, D. V. Hertem, L. Zhang, and H.-P. Nee, "Prospects and challenges of future HVDC SuperGrids with modular multilevel converters," in 2011 14th European Conf. Power Electron. and Applicat. (EPE'11 ECCE Europe), Aug. 2011, pp. 1-10.

[2] N. Ahmed, S. Norrga, H.-P. Nee, A. Haider, D. V. Hertem, L. Zhang, and L. Harnefors, "HVDC supergrids with modular multilevel converters - The power transmission backbone of the future," in Int. Multi-Conf. Systems, Signals and Devices, Mar. 2012, pp. 1-7.

[3] A. Lesnicar and R. Marquardt, "An innovative modular multilevel converter topology suitable for a wide power range," in IEEE Bologna Power Tech Conf. Proc., vol. 3, June 2003.

[4] — "A new modular voltage source inverter topology," in 2003 10th European Conf. Power Electron. and Applicat. (EPE'03 ECCE Europe), Sept. 2003.

[5] R. Marquardt and A. Lesnicar, "New concept for high voltage - modular multilevel converter," in IEEE PESC 2004, Aachen, Germany, June 2004.

[6] M. Glinka and R. Marquardt, "A new ac/ac multilevel converter family," in IEEE Trans. Ind. Electron., vol. 52, no. 3, June 2005, pp. 662-669. 
[7] J. Häfner and B. Jacobson, "Proactive hybrid HVDC breakers - A key innovation for reliable HVDC grids," in The electric power system of the future - Integrating supergrids and microgrids International Symposium in Bologna, Italy, 2011.

[8] S. Norrga, X. Li, and L. Ängquist, "Converter topologies for hvdc grids," in IEEE Int. Energy Conf. (ENERGYCON), May 2014, pp. 1554-1561.

[9] R. Marquardt, "Modular multilevel converter topologies with dc-short circuit current limitation," in 2011 8th Int. Conf. Power Electron. and ECCE Asia (ICPE 2011-ECCE Asia), May 2011, pp. 1425-1431.

[10] M. M. C. Merlin and T. C. Green, "Cell capacitor sizing in multilevel converters: cases of the modular multilevel converter and alternate arm converter," in IET Power Electron., vol. 8, no. 3, 2015, pp. 350-360.

[11] S. Heinig, K. Ilves, S. Norrga, and H.-P. Nee, “On energy storage requirements in alternate arm converters and modular multilevel converters," in 2016 18th European Conf. Power Electron. and Applicat. (EPE'16 ECCE Europe), Sept. 2016.

[12] K. Ilves, L. Bessegato, L. Harnefors, S. Norrga, and H.-P. Nee, "Semi-full-bridge submodule for modular multilevel converters," in 2015 9th Int. Conf. Power Electron. and ECCE Asia (ICPE 2015-ECCE Asia), June 2015, pp. 1067-1074.

[13] M. Rabuffi and G. Picci, "Status quo and future prospects for metallized polypropylene energy storage capacitors," in IEEE Trans. Plasma Sci., vol. 30, no. 5, Oct. 2002, pp. 1939-1942.

[14] HITACHI, “Datasheet IGBT MODULE MBN800E33E,” 2013.

[15] F. Dijkhuizen and S. Norrga, "Fault tolerant operation of power converter with cascaded cells," in 2011 14th European Conf. Power Electron. and Applicat. (EPE'11 ECCE Europe), Aug. 2011, pp. 1-8.

[16] M. Billmann, D. Malipaard, and H. Gambach, "Explosion proof housings for IGBT module based high power inverters in HVDC transmission application," in Proc. 2009 PCIM Europe Conf., 2009, pp. 352-357.

[17] Vishay, "Datasheet ESTAdry-DC-Capacitor TYPE DCMKP 2.65/4.0mF," 2008. 